\title{
Development of Pulsed Solid-State Generators of Millimeter and Submillimeter Wavelengths Based on Multilayer GaAs/AlGaAs Heterostructures
}

\author{
V.A. Gergel, N.M. Gorshkova, R.A. Khabibullin, P.P. Maltsev, \\ V.S. Minkin, S.A. Nikitov, A.Yu. Pavlov, V.V. Pavlovskiy and
} A.A. Trofimov

\begin{abstract}
This paper presents the results of research of electrical characteristics features of multibarrier AlxGa1-xAs/GaAs heterostructures with tunnel-nontransparent potential barriers. Briefly described constructive-technological features fabricated using molecular beam epitaxy. We measured the quasi-static current-voltage characteristics of test items by electric pulses of duration $10^{-6} \mathrm{~s}$ and a duty cycle of 103 . Characteristics observed with a strong section of the negative differential resistance in the current range of several tens of milliamperes. It is proposed to use this effect for the generation of terahertz electromagnetic radiation. The theoretical interpretation of the observed phenomena on the basis of quasi-hydrodynamic theory of electron drift is briefly stated .
\end{abstract}

Keywords Terahertz pulse generator - Multi barrier heterostructure Semiconductor $\cdot$ Microwave radiation

Investigated heterostructure made by molecular beam epitaxy on a conductive substrate of GaAs, is a system of alternating layers of high-alloy $\left(\mathrm{Nd} \sim 10 \mathrm{e} 18 \mathrm{~cm}^{-3}\right) \mathrm{GaAs}$ and barrier layers Al0.25Ga0.75As, about $50 \mathrm{~nm}$ thick. The first and last barrier layers have a reduced content of Al $x=0.125$, that

\footnotetext{
V.A. Gergel · N.M. Gorshkova (ه) · V.S. Minkin · S.A. Nikitov ·

V.V. Pavlovskiy

Institution of Russian Academy of Sciences, Kotelnikov Institute

of Radio Engineering and Electronics of RAS, Moscow, Russia

e-mail:vgergel@mail.ru

S.A. Nikitov

e-mail: nikitov@cplire.ru
}

R.A. Khabibullin · P.P. Maltsev · A.Yu. Pavlov · A.A. Trofimov

Institution of Russian Academy of Sciences, Institute of Ultra-High Frequency

Semiconductor Electronics of RAS, Moscow, Russia 


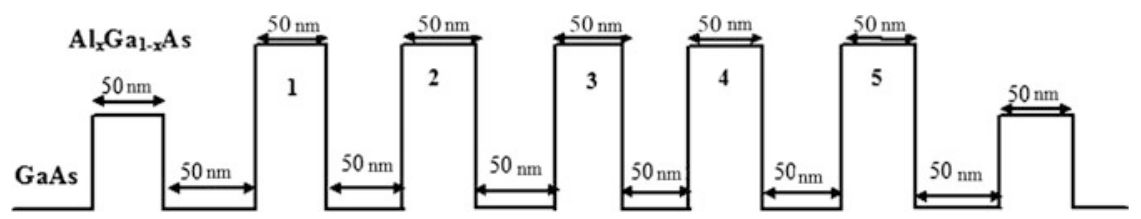

Fig. 1 The energy profile of the conduction band edge of the investigated heterostructures

is, a lower height of energy compared with the other (see the energy diagram on Fig. 1). Then by wet etching on the plate-shaped system quasicylindrical mesa element as a diameter of about $10-15 \mu \mathrm{m}$, the side surface of which is isolated $\mathrm{CVD} \mathrm{SiO}_{2}$ deposition. After opening corresponding windows on top of the each element was formed and an ohmic contact of ( $\mathrm{Au}$ ) metallization. The corresponding metal contact has been let down and to the vicinity of the test element base.

\section{Quasi-Static Current-Voltage Characteristics}

Quasi-static current-voltage characteristics of test items were measured in pulsed current sequence mode, increasing the amplitude of the duration of $1 \mu$ s and a repetition rate of $1 \mathrm{kHz}$. At the same characteristics, a number of them showed a distinct region of negative differential resistance in the region of relatively high current values $<50 \mathrm{~mA}$ and on the stress scale Section 1.5-2 B (Fig. 2).

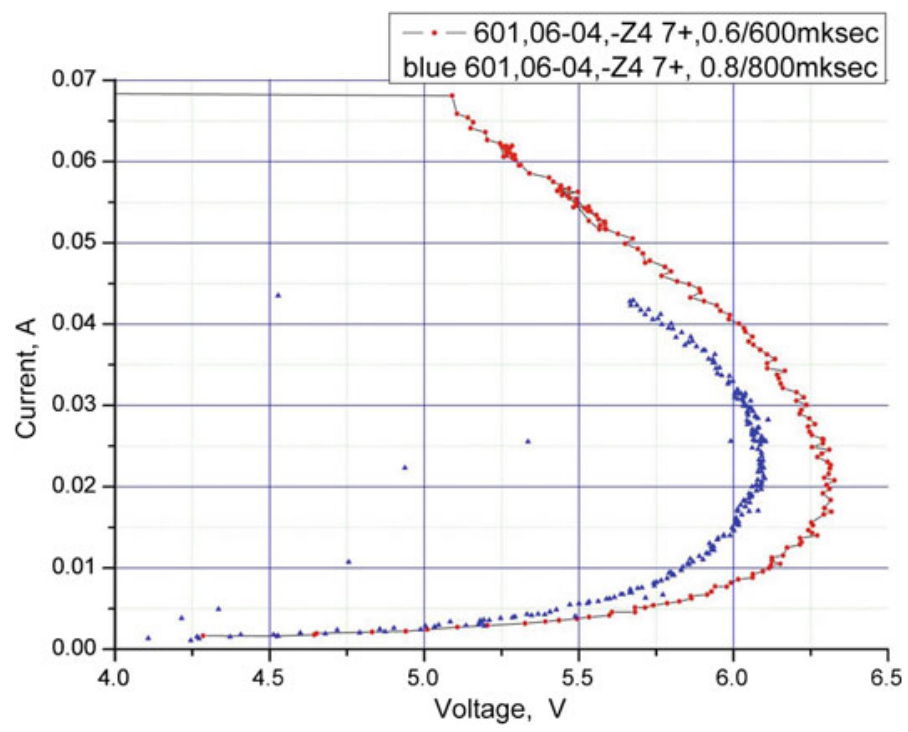

Fig. 2 Experimental quasi-static CVC of test element with NDR 
We believe that the observed NDR responds the so-called effect thermo-injective instability, which is a qualitative explanation is that in these heterostructures applied voltage is concentrated mainly in the undoped barrier layers. The adjacent layers of heavily doped GaAs electric field are relatively small. The electrons from the GaAs valleys overcome the corresponding energy barriers by thermo-injection. In this case, the appropriate drift process that ensures the continuity of the current takes place in the so-called non-local energy balance, when the electrons are heated in the undoped barrier layers in which the concentrated high electric field, and cooled in the adjacent heavily doped GaAs layers with a weak electric field. At the same time, a certain portion of the electron heat flux increases with increasing current reaches the next energy barrier and enhances injection of electrons through the barrier into the next GaAs valley. Therefore, to maintain a constant electron current should be reduced electron heating in the previous barrier layer that is provided by a corresponding decrease of incident voltage, which causes the S-shape of the resulting current-voltage characteristics with a strong region of the negative differential resistance. Despite the marked non-locality, the average Joule energy is transformed into a crystal lattice, resulting in a corresponding heating of the entire sample. That is why to reduce the temperature of the sample electrical device is powered by microsecond short current pulses with a repetition frequency of about $1 \mathrm{kHz}$. For a more convincing interpretation of the results should refer to the so-called quasi-hydrodynamic theory of electron drift (thermal model), which includes a Poisson equation, the equation of continuity of the electron beam (1-2):

$$
\frac{\mathrm{d} \varphi}{\mathrm{d} x}=\frac{q}{\varepsilon \varepsilon_{0}}\left(N_{d}-n\right)
$$

and

$$
\frac{\partial j_{n}}{\partial x}=0
$$

and the continuity of electron temperature flow (3):

$$
\frac{\mathrm{d} j_{T}}{\mathrm{~d} x}=j_{n} \frac{\mathrm{d} \varphi}{\mathrm{d} x}-n \frac{T-T_{0}}{\tau}
$$

in the formulation of Stratton [5, P. 453], and characterized in that in it summands with electrostatic potential $\varphi$ supplemented summand with zone quasipotential $\varphi_{c}=-E_{c}(x) / q$, where $E_{c}(x)$ - the edge of the conduction band, varying with the coordinate in accordance with a change in composition.

The system (1-3) discloses the expression for the flow of the electron density and the electron temperature (4-5): 


$$
j_{n}=\mu n\left[\frac{\mathrm{d}\left(\varphi+\varphi_{c}\right)}{\mathrm{d} x}-(1-\alpha) \frac{\mathrm{d} T}{\mathrm{~d} x}\right]-\mu T \frac{\mathrm{d} n}{\mathrm{~d} x}
$$

and

$$
j_{T}=\left(\frac{5}{2}-\alpha\right)\left(-\mu n T \frac{\mathrm{d} T}{\mathrm{~d} x}+j_{n} T\right)
$$

written on the assumption the power dependence of the kinetic coefficients of mobility and the relaxation time of the electron temperature $T(6-7)$ :

$$
\begin{gathered}
\mu=\mu_{0}\left(\frac{T_{0}}{T}\right)^{\alpha} \\
\tau=\tau_{0}\left(\frac{T}{T_{0}}\right)^{1-\alpha}, \quad 0<\alpha<1
\end{gathered}
$$

Solutions of this system of equations obtained by numerical simulation methods in a certain range of parameters clearly demonstrated S-shaped resulting currentvoltage characteristics, indicative of an electric instability considered heterostructures $[1$, P. $453 ; 2$, P. 1075]. Note that when $\alpha=1$, the equation of the electron temperature is linear flow continuity in heavily doped GaAs valleys, allowing you to build and relevant analytical solutions for the electron temperature. As expected, diffusion (caused by thermal conductivity) component of the heat flux decreases exponentially in the direction of electron drift in the valley with a decrement decreases with increasing current density, which increases the heat transfer from the previous warming up electrons barrier to the next, making it easy to thermionic bridging and hence reduces the need for heating in the previous barrier, i.e., reduces the voltage drop across it. Note that such an analytical model, [3, P. 481; 4, P. 83], has been widely used in the calculation of the expected characteristics of the test heteroelements, showing a characteristic range of currents and voltages NDR area. Figure 3 shows typical resulting curves obtained under the effect of greatly simplified analytical model.

Previously, we could not consider the structure with uneven barriers and that we strongly limited. In particular, a half-height front barrier empirically necessary to do in order to provide preheating. Therefore, the development of the above model, we abandoned the cyclic boundary conditions and constructed an algorithm sequential solution to the problem of thermionic, starting with the first input of the unit cell comprising a contact layer high-alloy material and the adjacent potential barrier. Optimization was necessary to construct an algorithm that takes into account the uneven barriers and differences valleys (8)-(11). The results of this calculation produce a flow of electrons into the second cell, which is a boundary condition for the character to overcome the electron beam of the second cell and so on. This algorithm allows to consider the structure with different thicknesses of the barrier 


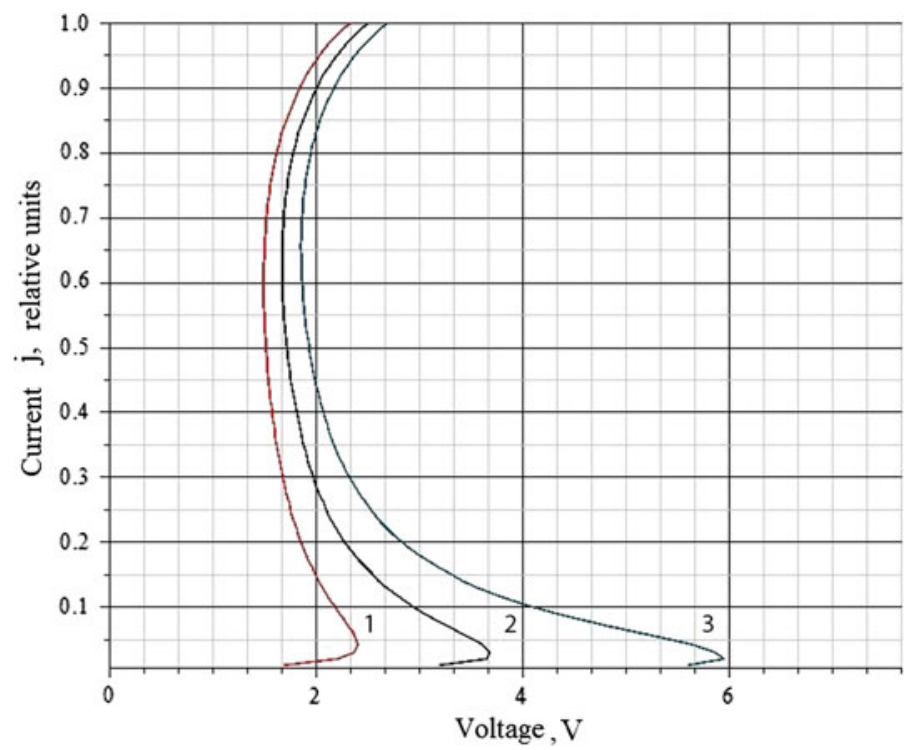

Fig. 3 Typical results CVC modeling in the framework of the analytical model

layers and high valleys. It turns out that the whole relaxation takes at least four barriers, despite the fact that we have to undergo substantial thickness of the layers, subject to the criterion of quality - minimizing heat release. This achieves sufficiently significant value UDF, which provides the ability to generate terahertz radiation at a certain partial minimize stress on the energy barriers, by varying the thickness of the individual layers and the selection of the ratio of the heights of the energy barriers. Compile the above model in the algorithm above formulas (1)-(7) were supplemented by the resulting expression expressing the electron temperature flow in the valleys, allowing to determine the voltage at the next barrier is needed to maintain the value of a given current, bearing in mind that before preceding the first barrier is no barrier, and therefore the adjoining contact layer relatively low electron temperature.

$$
\begin{gathered}
T_{0}=\frac{1}{1-\frac{2}{3} j^{2}} \\
V_{i}^{+}=j d^{+} T_{i} \exp \left(\frac{\Delta}{T_{i}}\right) \\
\tilde{V}_{N}=\sum_{i=0}^{N} V_{i} \mathrm{e}^{-(N-i) K d_{-}}
\end{gathered}
$$




$$
T_{N}=T_{0}\left(1+\frac{2}{3} j \tilde{V}_{N} K e^{-K d_{-}}\right)
$$

\section{Conclusions}

Characteristics were observed with a strong section of the negative differential resistance in the current range of several tens of milliamperes. It is proposed to use this effect for the generation of terahertz electromagnetic radiation. The theoretical interpretation of the observed phenomena on the basis of quasi-hydrodynamic theory of electron drift is briefly stated.

Acknowledgments Researches are carried out (conducted) with the financial support of the state represented by the Ministry of Education and Science of the Russian Federation. Agreement (contract) no. 14.607.21.0141 27. October 2015. Unique project Identifier: RFMEFI60715X0141.

\section{References}

1. Gergel, V.A., et al.: Semiconductors, 39, 453-455(2005)

2. Gergel, V.A., et al.: Semiconductors, 39, 1075-1079 (2005)

3. Gergel, V.A., et al.: Semiconductors, 48, 481-486 (2014)

4. Gergel, V.A., et al.: Technical Physics, 85, 83-86 (2015)

5. Stratton, R.: Diffusion of hot and cold electrons in semiconductor barriers. Phys. Rev. 126(6), 2002 (1962)

Open Access This chapter is licensed under the terms of the Creative Commons Attribution 4.0 International License (http://creativecommons.org/licenses/by/4.0/), which permits use, sharing, adaptation, distribution and reproduction in any medium or format, as long as you give appropriate credit to the original author(s) and the source, provide a link to the Creative Commons license and indicate if changes were made.

The images or other third party material in this book are included in the book's Creative Commons license, unless indicated otherwise in a credit line to the material. If material is not included in the book's Creative Commons license and your intended use is not permitted by statutory regulation or exceeds the permitted use, you will need to obtain permission directly from the copyright holder. 